\title{
Particle Production and Fragmentation at HERA
}

Grażyna Nowak ${ }^{\mathrm{a} *}$

on behalf of the H1 and ZEUS Collaborations

${ }^{a}$ Henryk Niewodniczański Institute for Nuclear Physics PAN

ul. Radzikowskiego 152, 31-342 Kraków, Poland

Recent results from the $\mathrm{H} 1$ and ZEUS collaborations on particle production in $e p$ scattering at HERA are presented. These results include measurements of the scaled momentum distribution, the charge asymmetry and the $p_{T}$ spectra of charged hadrons, the measurement of $K_{S}^{0}$ production and the study of charm quark fragmentation into $D^{* \pm}$ mesons. The data are compared to Monte Carlo expectations, to predictions of NLO QCD calculations and if possible, to results from $e^{+} e^{-}$annihilation and $p \bar{p}$ collision experiments.

\section{INTRODUCTION}

The study of particle production and fragmentation processes in $e p$ collisions allows detailed investigations of the non-perturbative regime of Quantum Chromodynamics (QCD). The HERA measurements complement the results obtained at other experiments and make it possible to test the universality of QCD in the particle formation process. The experiments $\mathrm{H} 1$ and ZEUS have taken data at the ep collider HERA in the years 1992 to 2007. Each experiment has collected data corresponding to an integrated luminosity of $\mathcal{L}_{\text {int }} \approx 0.5 \mathrm{fb}^{-1}$. The results presented here have been obtained in two different regimes of the $e p$ scattering defined by the four momentum transfer squared $Q^{2}$ of the exchanged photon: photoproduction $(\gamma p)$ having $Q^{2} \approx 0 \mathrm{GeV}^{2}$ and deep inelastic scattering (DIS) for which $Q^{2} \geq 2 \mathrm{GeV}^{2}$ is required here.

\section{CHARGED PARTICLE PRODUC- TION}

\subsection{Scaled momentum spectra of charged particles in $\gamma p$ and DIS}

Scaled momentum spectra are studied in the current region of the Breit frame. The scaled momentum variable $x_{p}$ is defined as $x_{p}=2 p_{h} / Q$,

*supported by DESY, Hamburg, Germany and Polish Ministry of Science and Higher Education, grant DPN/N168/DESY/2009
ZEUS

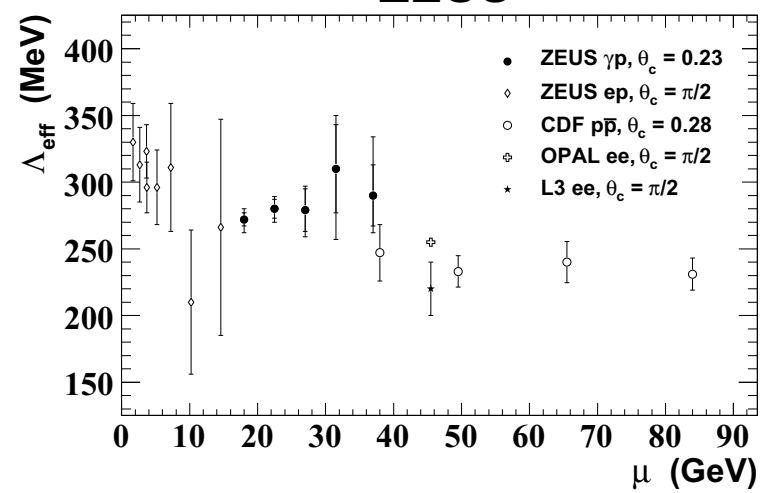

Figure 1. $\Lambda_{\text {eff }}$ as a function of $\mu$, where $\mu$ denotes the energy scale for the specific process.

where $p_{h}$ is the momentum of a charged particle in the Breit frame. The momentum spectra of charged hadrons can be predicted by perturbative QCD using the modified leading log-approximation (MLLA) and assuming local parton-hadron duality (LPHD). The MLLA cutoff parameter, $\Lambda_{e f f}$ and the LPHD constant normalisation scaling factor $\kappa^{c h}$, are expected to be process independent. The ZEUS collaboration measured scaled momentum distributions in dijet events in photoproduction [1]. The distributions of the variable $\xi=\ln \left(1 / x_{p}\right)$ are measured in five bins of the jet energy, $E_{j e t}$. The peak po- 


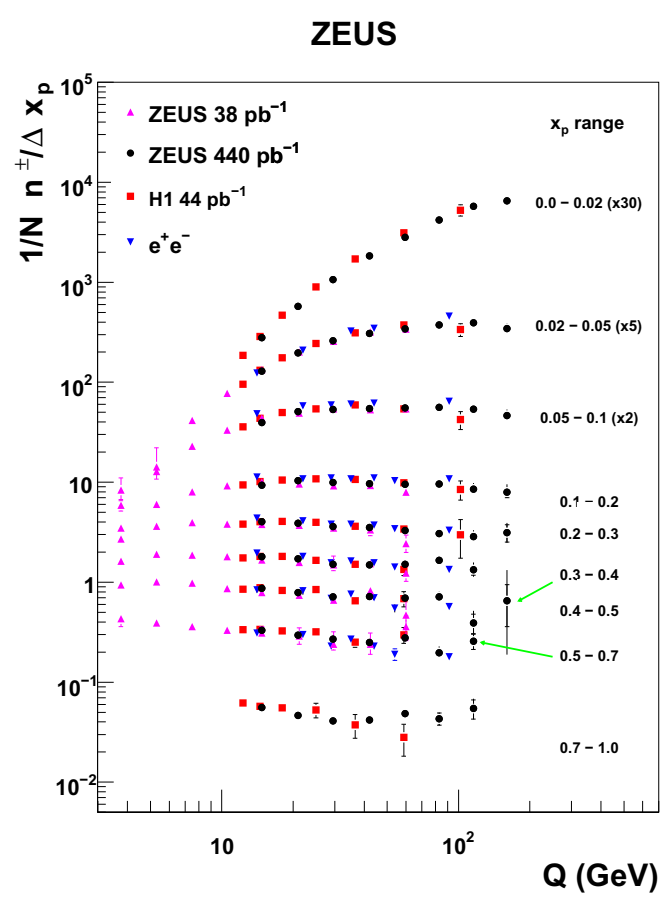

Figure 2. The normalized spectrum of charged particles as a function of $Q$ in $x_{p}$ bins. with width $\Delta x_{p}$. Also shown are data from the $\mathrm{H} 1$

sitions, $\xi_{\text {peak }}$ of the $\xi$ distributions are extracted and values of $\Lambda_{\text {eff }}$ are obtained by fitting the $\xi_{\text {peak }}$ data to the MLLA predictions of the energy scale dependence. In Figure 1 the values of $\Lambda_{\text {eff }}$ are shown as a function of the energy scale and compared to the previous results from ZEUS $e p$ DIS data [2], from $e^{+} e^{-}[3,4]$ and $p \bar{p}[5]$ experiments. The value of the LPHD parameter $\kappa_{c h}$ was extracted from the shape of the $\xi$ distributions. The obtained value of $\kappa_{c h}=0.55$ is consistent with the results published by the CDF collaboration [5]. The data support the universality of the $\Lambda_{e f f}$ and $\kappa_{c h}$ parameters.

The $Q^{2}$ evolution of the scaled momentum distribution is measured by the ZEUS in the kinematic region $10<Q^{2}<41000 \mathrm{GeV}^{2}$ [6]. The data are presented in Figure 2 and compared with $e^{+} e^{-}$data and with previous $\mathrm{H} 1$ measurements [7]. The different measurements are in reasonable agreement and thereby support the con-

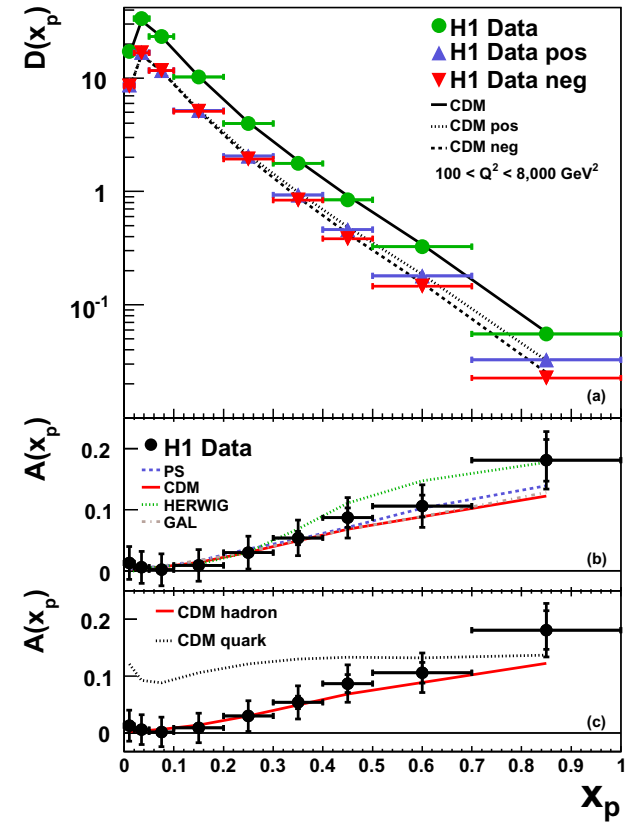

Figure 3. The measured normalised distributions of the scaled momenta for all charged particles and for positively (pos) and negatively (neg) charged particles (a), and the charge asymmetry as a function of $x_{p}(\mathrm{~b}, \mathrm{c})$. The data are compared to predictions from different Monte Carlo models.

cept of quark fragmentation universality. The data show clear scaling violation, i.e. the number of charged particles increases/decreases with $Q^{2}$ at low/high $x_{p}$, respectively.

\subsection{Hadronic final state charge asymme- try in DIS}

The charge asymmetry in the hadronic final state in DIS in the range of $100<Q^{2}<8000 \mathrm{GeV}^{2}$ is measured by H1 [8]. The measurement is performed in the current hemisphere of the Breit frame.

The measured scaled momentum distribution $D\left(x_{p}\right)$ for all charged particles and separated for positive and negative particles is shown in Figure 3 together with the charge asymmetry $A\left(x_{p}\right)=\left(D^{+}\left(x_{p}\right)-D^{-}\left(x_{p}\right)\right) / D\left(x_{p}\right)$. The data are compared to the Monte Carlo (MC) models on the parton and hadron level. The observed charge asymmetry shows a strong dependence 
on $x_{p}$. This is consistent with the expectation that low $x_{p}$ region is dominated by fragmentation while high $x_{p}$ region is sensitive to the hard subprocesses and the asymmetry is directly related to the valence quark content of the proton. The MC models describe the magnitude and the $x_{p}$ dependence of the asymmetry well.

\subsection{Transverse momentum $p_{T}$ of charged particles at low $Q^{2}$}

The HERA collider gives access to small values of Bjorken $x$ of about $10^{-5}$. In this domain the description of the parton dynamics in the DGLAP scheme [9] may become inappropriate. The measurement of the transverse momentum distribution of hadrons in the final state is expected to carry information about the parton shower evolution. The measurement is performed

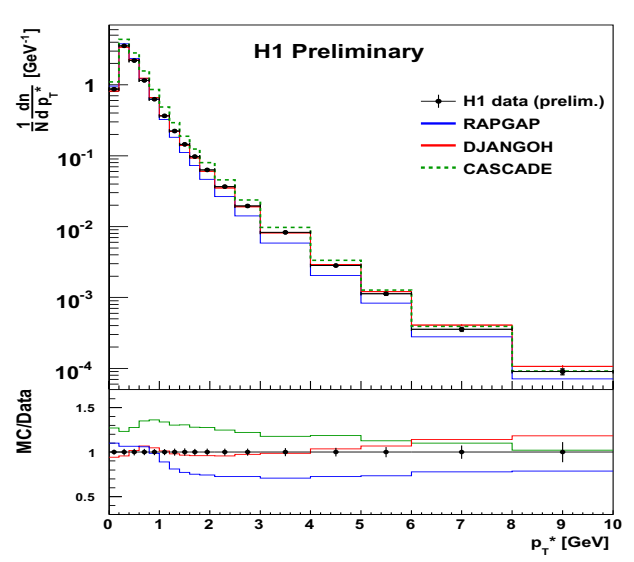

Figure 4. The measured $p_{T}^{*}$ spectrum of charged particles. The "MC/Data" ratios are shown for different $\mathrm{MC}$ predictions. For comparison the data points are put to one.

in DIS for $2<Q^{2}<100 \mathrm{GeV}^{2}$ in the hadronic center of mass system in the region of pseudorapidity, $1.5<\eta^{*}<2.5$. The measured $p_{T}^{*}$ spectrum of charged particles is shown in Figure 4 . The data are well described over the whole $p_{T}^{*}$ spectrum by the predictions of the DJANGOH MC program [10] in which parton showers evolve according to the colour dipole model (CDM) [11]. The RAPGAP model [12] based on DGLAP evolution

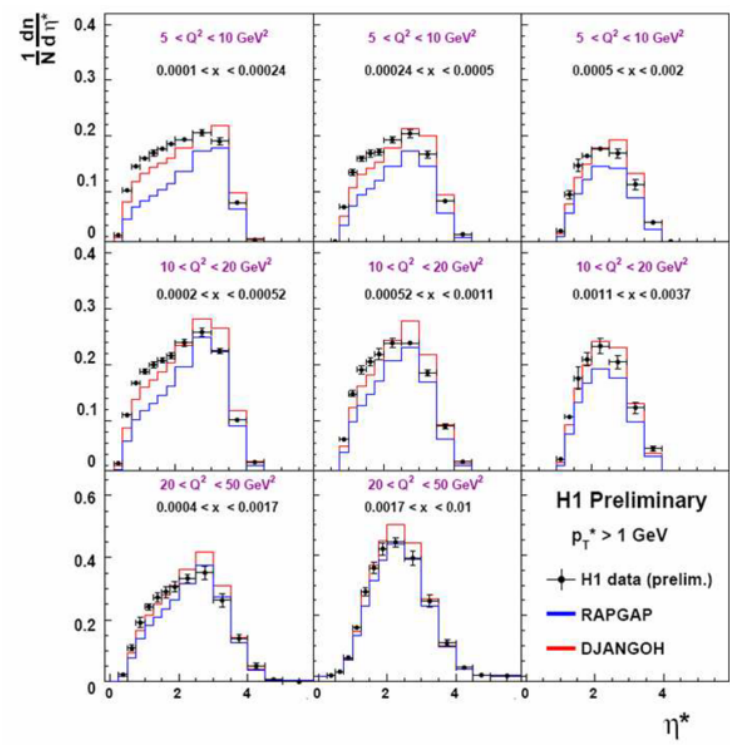

Figure 5. The average multiplicity of charged particles as a function of $\eta^{*}$ for $p_{T}^{*}>1 \mathrm{GeV}$ in bins of $Q^{2}$ and $x$. Data are compared with different $\mathrm{MC}$ predictions.

falls significantly below the data for $p_{T}^{*}>1 \mathrm{GeV}$. The prediction from CASCADE [13] based on CCFM equations [14] fails to describe the data in shape and normalisation. The measured flow of charged particles with $p_{T}^{*}>1 \mathrm{GeV}$ as a function of $\eta^{*}$ is shown in Figure 5 in bins of $x$ and $Q^{2}$. The data are well described over the full kinematic range by DJANGOH while RAPGAP is below the data especially at small $Q^{2}$. These observations give evidence that the colour dipole model is more appropriate for describing the parton shower evolution in this kinematic region.

\section{STRANGENESS PRODUCTION in DIS at HIGH $Q^{2}$}

Strange quarks can be produced perturbatively by the boson-gluon fusion. The proton parton densities and the non-perturbative colour field fragmentation are additional sources of strange quarks. The strange hadrons are then produced in the hadronisation process and by the weak decays of heavy flavoured hadrons. Precise knowledge about the suppression of strange quark-pair 
creation relative to lighter flavours, defined by the parameter $\lambda_{s}$ is important for phenomenological models of the fragmentation process. The H1 collaboration has investigated $K_{S}^{0}$ production in DIS in the kinematic range of $125<Q^{2}<20000 \mathrm{GeV}^{2}$. Single differential cross-sections of $K_{S}^{0}$ production are measured as a function of kinematic variables. As an example the $K_{S}^{0}$ production cross section as a function of $Q^{2}$ is shown in Figure 6. A comparison of the measured single differential cross sections with predictions based on LO Monte Carlos shows a good overall agreement when using a strangeness suppression factor of $\lambda_{s}=0.286$.

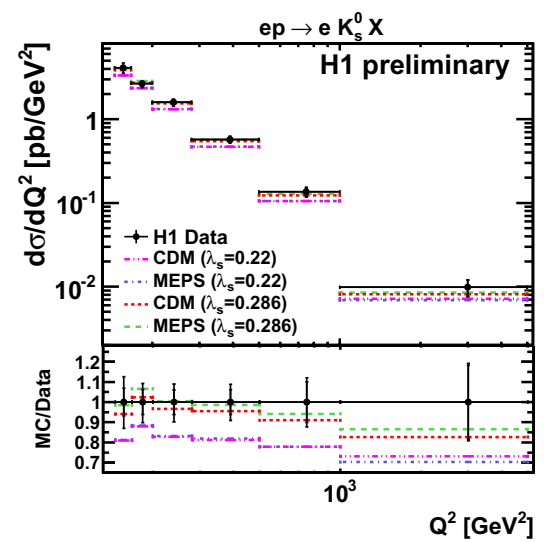

Figure 6. The differential production cross section for $K_{S}^{0}$ mesons. On the bottom of the figure the "MC/Data" ratios are shown for different MC predictions. The data points are put to one.

Similar conclusions can be drawn from the strange-to-light hadron ratio measurements. This value of $\lambda_{s}$ is in agreement with the value obtained from $e^{+} e^{-}$collisions supporting the universality of strangeness production.

\section{CHARM FRAGMENTATION into $D^{*}$ MESONS in $\gamma p$ and DIS}

The ZEUS collaboration measured the charm quark fragmentation function to $D^{*}$ mesons in $\gamma p$ [15]. The fragmentation function is measured using the variable $z=\left(E+P_{L}\right)_{D^{*}} / 2 E_{j e t}$ where $E$ is the energy of the $D^{*}$ meson and $P_{L}$ is the longitudinal momentum of the $D^{*}$ meson w.r.t. the axis of the associated jet of energy $E_{j e t}$. The

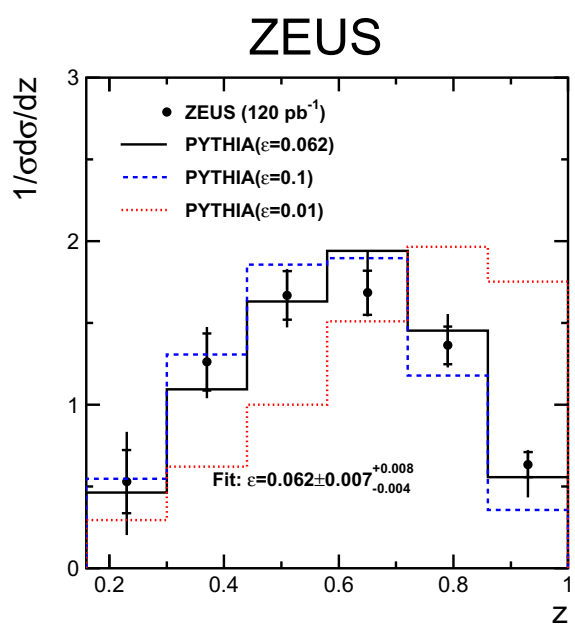

Figure 7. The normalised cross-section for the data compared with PYTHIA simulations with varying values of the parameter $\epsilon$. The result of fitting the MC to data is shown as a solid line.

transverse energy of the jet is required to be larger than $9 \mathrm{GeV}$. All quantities are given in the laboratory frame. The measured fragmentation function is compared to expectations from LO MC models and to NLO QCD calculations. Often used parametrisations of the nonperturbative fragmentation function are the Peterson function [16] and the Kartvelishvili function [17] having a single free parameter $\epsilon$ and $\alpha$, respectively. The PYTHIA simulations [18] using the Peterson function with varying $\epsilon$ parameter are compared with the data as shown in Figure 7 . The value of the $\epsilon=0.062$ extracted from fitting the MC to the data is consistent with the value of $\epsilon=0.05$ obtained from $e^{+} e^{-}$data within uncertainties. The data and the results of a NLO QCD calculation [19] with varying $\epsilon$ parameter are shown in Figure 8. The value of the $\epsilon=0.079$ is extracted from the fit of the predictions to the data. Within the consistent framework given by the PYTHIA model this value agrees with the value of the $\epsilon$ parameter determined in $e^{+} e^{-}$data [20].

The charm fragmentation function to $D^{* \pm}$ has been investigated by H1 in DIS [21]. Two different event samples are used for the measurement. In the first sample, referred to as " $D^{* \pm}$ 




Figure 8. The normalised cross-section for the data compared with calculation of NLO QCD (FMNR) with Peterson function. The result of fitting the MC to data is shown as a solid line.

jet sample", the presence of a jet with transverse energy $E_{T}^{*}>3 \mathrm{GeV}$ containing the $D^{* \pm}$ meson is required, similar to ZEUS studies in $\gamma p$. In the second sample, referred to as "no $D^{* \pm}$ jet sample", no such jet is allowed. The latter sample enables the investigation of charm quark fragmentation in a region close to the kinematic threshold of charm production. In this analysis the fragmentation variable is defined as $z_{\text {hem }}=\left(E^{*}+P_{L}^{*}\right)_{D^{*}} / \sum_{h e m}\left(E^{*}+P^{*}\right)$ where the sum in the denominator runs over all particles belonging to a suitably defined hemisphere containing the $D^{* \pm}$ meson as an approximation for the charm quark fragmenting to the $D^{* \pm}$ meson. All quantities are given in $\gamma^{*} p$ rest-frame. The normalised $D^{* \pm}$ meson differential cross section as a function of the fragmentation variable is used to extract optimal parameters for the Peterson and Kartvelishvili functions. Figure 9 shows the measured cross section as a function of $z_{h e m}$ for the $D^{* \pm}$ jet sample together with the predictions of the LO MC model using the Kartvelishvili function. The data have also been compared to expectations using the Peterson function. The extracted fragmentation parameter $\epsilon$ agrees with the value obtained from $e^{+} e^{-}$data. Together

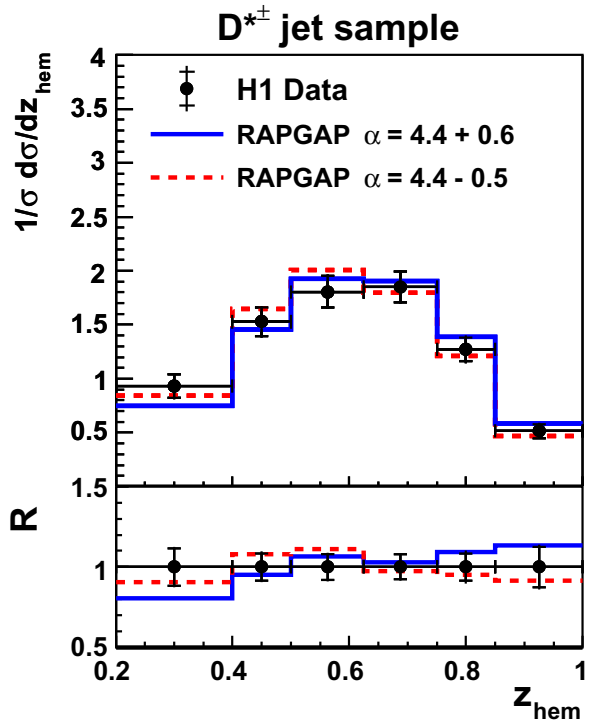

Figure 9. The normalised $D^{* \pm}$ meson crosssection for the $D^{* \pm}$ jet sample, as a function of $z_{\text {hem }}$. The data are compared to the predictions of LO MC program with Kartvelishvili function. The full and dashed lines indicate a variation of the fragmentation parameter by $\pm 1 \sigma$ around the best fit value of $\alpha$. The ratio $\mathrm{R}=\mathrm{MC} /$ data is shown for the data points put to $\mathrm{R}=1$.

with the findings in $\gamma p$ this result supports the hypothesis of universality of charm quark fragmentation. The fragmentation parameters fitted to the no $D^{* \pm}$ jet sample, however, are found to be significantly different from those for the $D^{* \pm}$ jet sample. As shown in Figure 10 the RAPGAP prediction using the fragmentation parameter obtained from the $D^{* \pm}$ jet sample is not able to describe these data. The same conclusions are obtained when comparing the data with the NLO calculations as implemented in the HVQDIS program [22]. This result indicates that our current modelling of charm fragmentation is not good enough to describe the data in the full region of phase space in $e p$ scattering.

\section{CONCLUSIONS}

Particle production has been studied in photoproduction and deep-inelastic $e p$ scattering at 


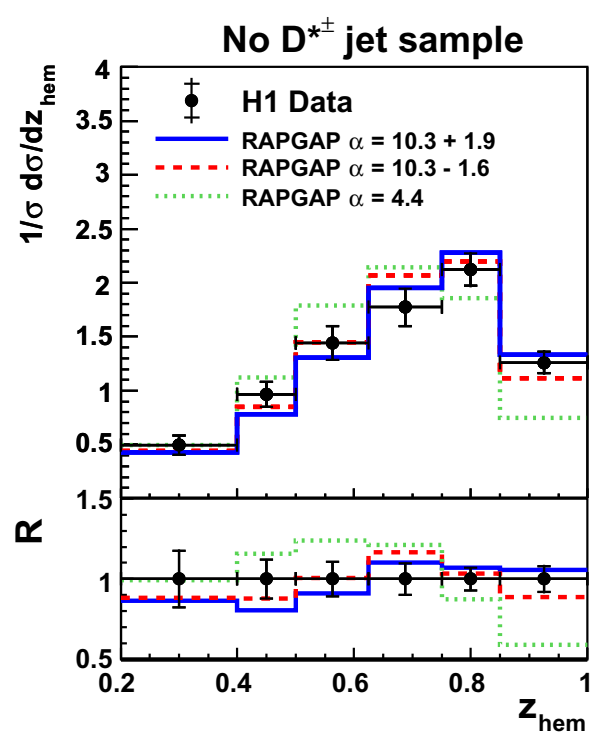

Figure 10. The normalised $D^{* \pm}$ meson crosssection as a function of $z_{\text {hem }}$ for the no $D^{* \pm}$ jet sample. The dotted line shows the prediction of $\mathrm{LO} \mathrm{MC}$ with the fragmentation parameter $\alpha=4.4$ extracted from the $D^{* \pm}$ jet sample. More details in caption of the Figure 9.

HERA. The parameters extracted from the measured scaled momentum distributions support the concept of quark fragmentation universality in $e p$, $e^{+} e^{-}$and $p \bar{p}$ collisions. The measured charge asymmetry of the hadronic final state at large fractional momentum is consistent with the expectation from the valence quark content of the proton. The charged particle spectra at relatively high hadron's transverse momentum give information about the parton shower evolution. $K_{S}^{0}$ production in DIS at high $Q^{2}$ are best described by using a value of $\lambda_{s}$ as determined from $e^{+} e^{-}$ data supporting the assumption of the universality of strangeness contribution in the hadronisation in $e p$ scattering and $e^{+} e^{-}$annihilation. Results on charm fragmentation to $D^{* \pm}$ mesons have been presented. The fragmentation parameter values obtained from events significantly above the charm quark production threshold are found to agree with measurements from $e^{+} e^{-}$annihilation. However it is not possible to get a consistent description of the charm quark fragmen- tation into $D^{* \pm}$ mesons over the full phase-space down to the kinematic threshold in ep scattering.

\section{REFERENCES}

1. S.Chekanov et al. [ZEUS Collaboration], JHEP 0908 (2009) 077.

2. M.Derrick et al. [ZEUS Collaboration], Z.Phys. C67 (1995) 93.

3. M.Z.Akrawy et al. [OPAL Collaboration], Phys. Lett. B247 (1990) 617.

4. B.Adeva et al. [L3 Collaboration], Phys. Lett. B259 (1991) 199.

5. D.E.Acosta et al. [CDF Collaboration], Phys. Rev. D68 (2003) 012003.

6. H.Abramowicz et al. [ZEUS Collaboration], JHEP 1006 (2010) 009.

7. F.D.Aaron et al. [H1 Collaboration], Phys. Lett. B654 (2007) 148.

8. F.D.Aaron et al. [H1 Collaboration], Phys. Lett. B681 (2009) 125.

9. V.Gribov and L.Lipatov, Sov. J. Nucl. Phys. 15 (1972) 575; G.Altarelli and G.Parisi, Nucl. Phys. B126 (1977) 298; Y.L.Dokshitzer, Zh. Eksp. Teor. Fiz.73 (1977) 1216.

10. K.Charchula et al. Comp. Phys. Commun. 81 (1994) 381.

11. B.Andersson et al. Z. Phys. C43 (1989) 525.

12. H.Jung, Comp. Phys. Commun. 86 (1995) 147.

13. H.Jung, Comp. Phys. Commun. 143100 (2002) 143.

14. M.Ciafaloni, Nucl. Phys. B86 (1988) 49; S.Catani et al. Nucl. Phys. B336 (1990) 18.

15. S.Chekanov et al. [ZEUS Collaboration], JHEP 0904 (2009) 082.

16. C.Peterson et al. Phys. Rev. D27 (1983) 105.

17. V.G.Kartvelishvili et al. Phys. Lett. B78 (1978) 615.

18. T.Sjöstrand et al., Comp. Phys. Commun. 136 (2001) 238.

19. S.Frixione et al. Phys. Lett. B348 (1995) 633.

20. H. Albrecht et al. [ARGUS Collaboration], Z. Phys. C52(1991) 353.

21. F.D.Aaron et al. [H1 Collaboration], Eur. Phys. J. C59 (2009) 589.

22. B.W.Harris and J.Smith, Phys. Rev. D57 (1998) 2806. 